\title{
Thoracoscopic right upper lobectomy in a patient with situs inversus totalis
}

\author{
Piotr Gabryel, Magdalena Sielewicz, Dominika Zielińska, Cezary Piwkowski \\ Department of Thoracic Surgery, Poznan University of Medical Sciences, Poznan, Poland
}

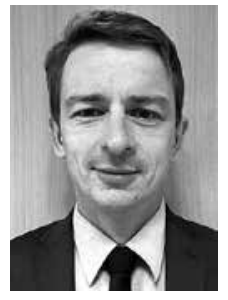

Kardiochir Torakochir Pol 2021; 18 (1): 62-63

Situs inversus totalis (SIT) is a rare congenital anomaly with an incidence ratio of $1: 4,000-1: 60,000$ births. It is characterised by the dislocation of the internal organs to the contralateral side of the body in relation to the median plane [1]. Video-assisted thoracoscopic surgery (VATS) is the recommended approach for the treatment of earlystage non-small cell lung cancer (NSCLC) [2, 3]. Variations in the lung anatomy of patients with SIT may complicate the resection of the lung, particularly when performed using a VATS approach.

A 65-year-old male with SIT was referred for the treatment of a tumour in the right upper lobe of the lung. His medical history revealed arterial hypertension, chronic obstructive pulmonary disease, and coronary heart disease.

Computed tomography (CT) of the chest revealed a 4.9 $\times 4.4-\mathrm{cm}$ mass in the right upper lobe of the lung. The right lung displayed a typical left lung anatomy, with 2 lobes and a long main bronchus passing below the aortic arch. A single atypical vein could be seen passing from the lin-

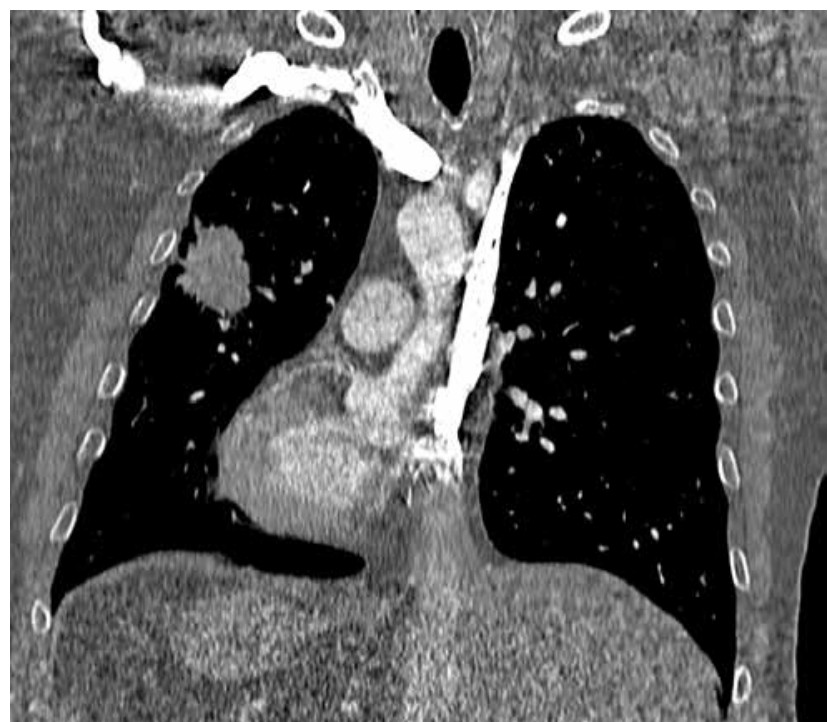

Figure 1. Computed tomography of the chest. A mass in the right upper lobe of the lung. Dislocation of the heart, great vessels, spleen, and liver to the contralateral side of the body gula to the right lower pulmonary vein. However, a lingular bronchus originated from the right upper lobe bronchus. The location of the internal organs, including heart, great vessels, liver and spleen, was typical of that in SIT (Figure 1). Positron emission tomography - computed tomography (PET-CT) using ${ }^{18} \mathrm{~F}-\mathrm{FDG}$ confirmed a $5.1 \times 4.5-\mathrm{cm}$ tumour with a standardised uptake value of 12.5 (Figure 2 ). Percutaneous CT-guided tumour biopsy established the diagnosis of squamous cell lung cancer. According to the $8^{\text {th }}$ edition of the TNM classification, the stage of the cancer was CT3, NO, MO.

The results of pulmonary function test were as follows: forced expiratory volume in $1 \mathrm{~s}$ (FEV1) 2.25 I (75\%) and vital capacity (VC) 3.57 I (95\%). Diffusion capacity for carbon monoxide was $9.82 \mathrm{mmol} / \mathrm{min} / \mathrm{kPa}$ (118\%). Apart from SIT, no other abnormalities were revealed using bronchoscopy, abdominal ultrasonography, electrocardiography, or echocardiography.

Surgery was performed under general anaesthesia. A left-sided double-lumen endotracheal tube was placed under the guidance of fibreoptic bronchoscopy, and single-lung ventilation was initiated. The 3-portal VATS approach was used. The well-developed, posterior part of the interlobar fissure was divided using LigaSure (Medtronic,

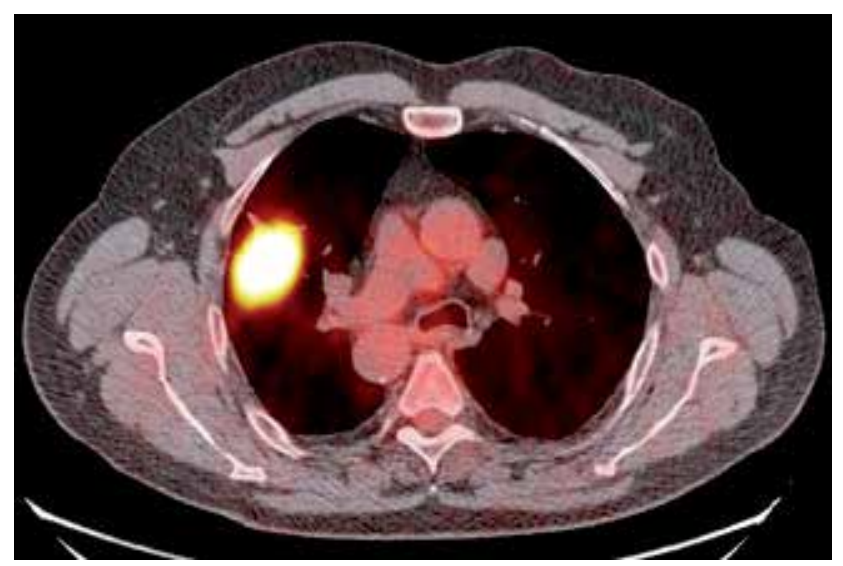

Figure 2. Positron emission tomography-computedtomography. Right upper lobe tumour with increased 18F-FDG uptake

Address for correspondence: Magdalena Sielewicz, Department of Thoracic Surgery, Poznan University of Medical Sciences, Poznan, Poland, e-mail: magda.sielewicz@gmail.com

Received: 14.09.2020, accepted: 6.01.2021. 
Minneapolis, MN, USA). Next, the common lingular artery was divided using an endostapler (Signia ${ }^{T M}$, Tri-Staple ${ }^{T M}$, Medtronic, Minneapolis, MN, USA). The anterior part of the interlobar fissure was divided using the endostapler at some distance from the branching lower pulmonary vein to avoid inadvertent hindrance to the lower lobe venous outflow. Subsequently, the segment 2 artery was divided using polymer clips (Click'aV ${ }^{\circledR}$, Grena, London, UK), and the upper pulmonary vein, segment $1 / 3$ artery and upper lobe bronchus were divided using endostaplers. The resected upper lobe was extracted in a sterile specimen bag. After re-inflation of the lung, part of the pulmonary parenchyma corresponding to segment 5 was found to be atelectatic and was subsequently removed. Paraaortic, aorto-pulmonary window, subcarinal, paraesophageal, hilar, and interlobar lymph nodes were removed. In the case of subcarinal nodes, the anterior approach was used; the standard posterior approach would have been less safe in this case, owing to the deep location of the carina and the proximity to the lower pulmonary vein, descending aorta, and oesophagus. Subsequently, a chest tube was introduced and connected to the digital drainage device with suction set at $-8 \mathrm{~cm} \mathrm{H}_{2} \mathrm{O}$. The patient's postoperative course was uneventful. His chest tube was removed on postoperative day (POD) 4, after transient air leak resolution, and he was discharged on POD 6. Final histopathology confirmed a diagnosis of squamous cell carcinoma (pT3, N0, R0, stage IIB).

To date, only a few cases of right-sided anatomical lung resection have been reported in patients with lung cancer and SIT. Only one such study referred to right upper lobectomy using VATS [4]. Although lung anatomy in patients with SIT is usually described as a 'mirror-image' of that in healthy individuals, some other minor and major anomalies may also occur. Using chest CT with intravenous contrast and 3-dimensional reconstructions is essential to identify abnormalities to effectively plan anaesthesiological and surgical procedures [5]. Intubation in patients with SIT may be difficult. Therefore, the bronchial tree should be evaluated using bronchoscopy prior to intubation. The introduction of a double-lumen endotracheal tube under endoscopic guidance may help in its proper positioning and prevent difficulties with lung ventilation.

\section{Acknowledgments}

The authors would like to thank Enago (www.enago. com) for the English language review.

\section{Disclosure}

The authors report no conflict of interest.

\section{References}

1. Wójcik J, Grodzki T, Bielewicz M, Wojtyś M, Kubisa B, Pieróg J, Wójcik N. Lung cancer in situs inversus totalis (SIT)-literature review. Adv Med Sci 2013; 58: 1-8.

2. Tane S, Nishio W, Okuma H, Ogawa H, Hokka D, Tane K, Tanaka Y, Uchino K, Yoshimura M, Maniwa Y. Operative outcomes of thoracoscopic lobectomy for non-small-cell lung cancer. Asian Cardiovasc Thorac Ann 2015; 23: 950-957.

3. Zhang Z, Zhang Y, Feng H, Yao Z, Teng J, Wei D, Liu D. Is video-assisted thoracic surgery lobectomy better than thoracotomy for early-stage non-small-cell lung cancer? A systematic review and meta-analysis. Eur J Cardiothorac Surg 2013; 44: 407-414.

4. Ye M, Shen J, Kong M, Lin J, Li X, Chen B. Video-assisted thoracoscopic surgery right upper lobectomy in a situs inversus totalis patient. J Thorac Dis 2017; 9: E1018-E1020.

5. Yoshida M, Hino H, Machida H, Hatakeyama N, Okano Y, Iwahara Y, Shinohara T, Oogushi F. ideo-assisted thoracic surgery lobectomy for lung cancer in a patient with complete situs inversus. Gen Thorac Cardiovasc Surg 2013; 61: 155-159. 